\title{
Gene Expression of Enzymes Comprising the Polyol Pathway in Various Rat Tissues Determined by the Competitive RT-PCR Method
}

\author{
Keiko Maekawa ${ }^{1, *}$, Tsuyoshi Tanimoto ${ }^{2}$ and Satoshi Okada ${ }^{2}$ \\ ${ }^{\prime}$ Division of Biochemistry and Immunochemistry, National Institute of Health Sciences, \\ 1-18-1 Kamiyoga, Setagaya, Tokyo 158-8501, Japan \\ ${ }^{2}$ Division of Drug Evaluation, National Institute of Health Sciences, 1-1-43 Hoenzaka, Chuo-ku, Osaka 540-0006, Japan \\ Received October 9, 2001 Accepted November 30, 2001
}

\begin{abstract}
The quantitative measurements of aldose reductase (AR) and sorbitol dehydrogenase (SDH) gene expression in various rat tissues were performed by the competitive reverse transcription-polymerase chain reaction (RT-PCR). AR mRNA was detectable in all tissues analyzed with pronounced differences in the amounts. SDH mRNA was most abundant in testes and liver, but was absent from lens. The estimation of the AR cDNA/SDH cDNA ratio showed that the relative abundance of SDH to AR differs among tissues. These results indicate that different tissues contain varying amounts of AR mRNA and SDH mRNA; that is, each tissue has its own polyol pathway activity.
\end{abstract}

Keywords: Aldose reductase, Sorbitol dehydrogenase, Diabetic complication

Aldose reductase (AR) (EC 1.1.1.21) and sorbitol dehydrogenase (SDH) (EC 1.1.1.14) constitute the polyol pathway, an alternative route of glucose metabolism. This pathway converts glucose to fructose via the sugar-alcohol intermediate sorbitol, using NADPH and $\mathrm{NAD}^{+}$as cofactors. A wealth of experimental data has indicated the involvement of the polyol pathway in the pathogenesis of diabetic complications such as neuropathy, retinopathy and nephropathy $(1,2)$. However, quantitative comparisons of each expression among tissues involved in the physiological and pathophysiological roles of these enzymes have not been fully studied yet. Furthermore there have been few studies on the relative abundance of SDH to AR in the tissues affected in diabetes. Systematic analyses of the expression of these two enzymes are essential because the flux through the polyol pathway and ensuing redox state changes, that is, changes in NADPH/NADP ${ }^{+}$or $\mathrm{NADH} / \mathrm{NAD}^{+}$ratios, are influenced by the balance of both enzyme activities. We have therefore developed a competitive reverse transcription-polymerase chain reaction (RT-PCR) assay system to simultaneously determine the mRNA levels of AR and SDH using a small amount of total RNA (3). The levels of AR and SDH gene expression were studied in various rat tissues including retina, lens and Schwann cells, in which the limited amounts of RNA

*Corresponding author. FAX: +81-3-3707-6950

E-mail: maekawa@nihs.go.jp are available.

Various tissues were dissected from 3 adult male Sprague-Dawley rats $(7-10$-week-old), except that ovary was taken from 3 female rats (10-week-old). Rats were allowed standard rat chow and water ad libitum, and their blood glucose fell within the range of $100-120 \mathrm{mg} / \mathrm{dl}$ before sacrifice. Schwann cells were isolated from the sciatic nerves as previously reported (4). Total RNA was isolated using RNAzol (TEL-TEST Inc.; Friendswood, TX, USA) and the yield was determined from the optical density at $260 \mathrm{~nm}$. Reverse transcription was performed with $0.5 \mu \mathrm{g}$ RNA using AMV reverse transcriptase (Promega, Madison, WI, USA), as described previously (3). Competitors for AR, SDH and glyceraldehyde-3-phosphate dehydrogenase (GAPDH) competitive RT-PCR, which are heterologous DNA fragments to which specific primer annealing sequences are added (PCR MIMIC), were created by two successive PCR amplifications (3). Serial dilutions of the AR, SDH or GAPDH PCR MIMIC were added to the reaction mixture containing a constant amount of cDNA sample. The amplification reactions were run under the following conditions: AR: denaturation at $95^{\circ} \mathrm{C}$ for $1 \mathrm{~min}$, annealing at $60^{\circ} \mathrm{C}$ for $1 \mathrm{~min}$ and extension at $72^{\circ} \mathrm{C}$ for $1 \mathrm{~min}$ for $25-30$ cycles; $\mathrm{SDH}$ : denaturation at $95^{\circ} \mathrm{C}$ for $1 \mathrm{~min}$, annealing at $60^{\circ} \mathrm{C}$ for $1 \mathrm{~min}$ and extension at $72^{\circ} \mathrm{C}$ for $1 \mathrm{~min}$ for $26-32$ cycles; GAPDH: denaturation at $95^{\circ} \mathrm{C}$ for $1 \mathrm{~min}$, annealing at $62^{\circ} \mathrm{C}$ for $1 \mathrm{~min}$ and extension at $72^{\circ} \mathrm{C}$ for $1 \mathrm{~min}$ for $22-25$ cycles. The number of cycles 
was adjusted for each tissue sample because the abundance of AR cDNA and SDH cDNA was quite different from tissue to tissue, so that we chose the minimum number of cycles sufficient to detect the amplified sample cDNA and PCR MIMIC by electrophoresis. The primers used for $\mathrm{AR}, \mathrm{SDH}$ and GAPDH competitive RT-PCR have been described previously (3). PCR products were electrophoretically separated, and the images of ethidium bromide-stained bands for amplified target fragments and their respective PCR MIMIC were digitized by the image gel documentation system (Image Master VDS; Amersham Pharmacia Biotech, Uppsala, Sweden). A linear regression analysis of log (signal cDNA / signal PCR MIMIC) versus $\log$ (known molecular quantities of the PCR MIMIC) was carried out for each reaction. The results of a typical experiment obtained from whole eye are shown in Fig. 1.

We confirmed the equal competitiveness of PCR MIMIC with target cDNA and the linear relationship between the amount of RNA subjected to reverse transcription and the calculated cDNA level (3). Under our experimental conditions, the amplification of AR, SDH and GAPDH was linear from $10^{-3}$ amol (lower limit of detection) to $0.5 \mathrm{amol}, 10^{-4}$ amol (lower limit of detection) to $0.1 \mathrm{amol}$, and $10^{-2}$ amol (lower limit of detection) to 3 amol of target cDNA, respectively.

The levels of AR mRNA in 17 different tissues are shown in Fig. 2A. AR mRNA was detectable in all tissues analyzed with pronounced differences in the amounts. The highest expression was observed in kidney medulla, followed by testes. Moderate levels of expression were observed in lung, ovary, adrenal gland, dorsal root ganglia, aorta, retina, whole eye, heart and lens. Lower levels of expression were observed in spinal cord, stomach and whole brain. The skeletal muscle (gastrocnemius muscle), kidney cortex and liver showed the lowest levels of AR mRNA, roughly 90 -fold less than in kidney medulla. In the cultured rat Schwann cells, AR mRNA was also expressed, and its level was 0.12 as AR cDNA / GAPDH cDNA ratio.

The abundant expression of AR in kidney medulla and testes further supports the involvement of this enzyme in important cellular functions, that is, in osmoregulation during the urinary concentration (5) and fructose production

\section{(A) AR}

\section{-Electrophoresis}
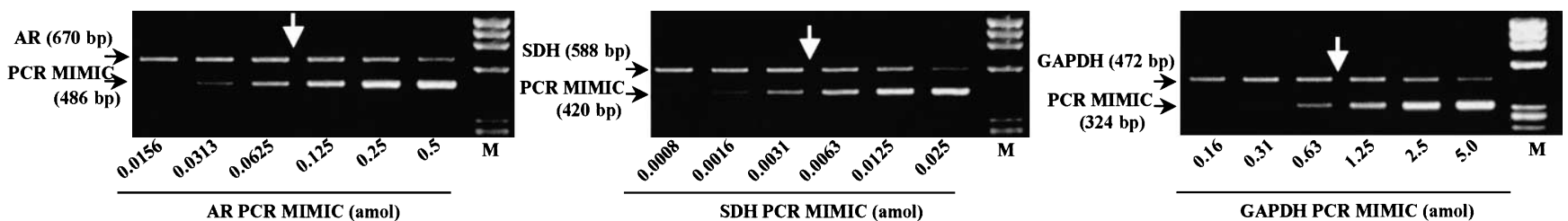

\section{-Regression plot}
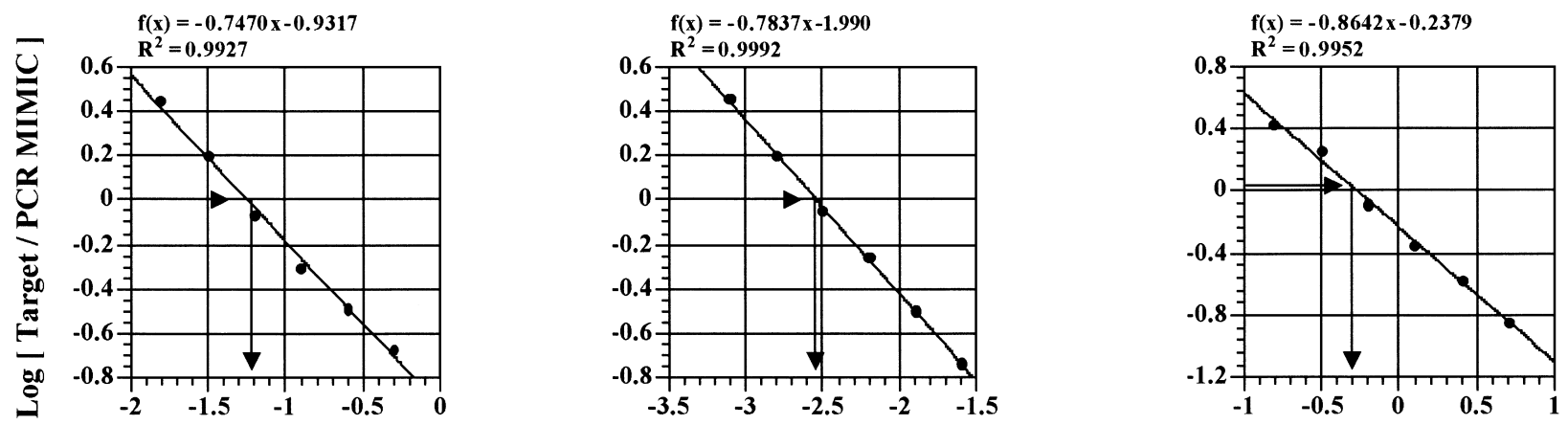

Log [ PCR MIMIC (amol) ]

Fig. 1. Competitive RT-PCR assay of AR (aldose reductase, A), SDH (sorbitol dehydrogenase, B) and GAPDH (glyceraldehyde3-phosphate dehydrogenase, C) mRNA in the whole eye from the adult rat. Upper panels show representative ethidium bromidestained gel electrophoresis profiles of the competitive RT-PCR products. Indicated by arrows are the points at which equal intensities of the target and competitor products were obtained by image analyses. M: DNA size marker ( $\phi x 174 H a e$ III digest). After being normalized for the size of the PCR products, the regression analyses of log (signal cDNA / signal PCR MIMIC) versus log (known molecular quantities of the PCR MIMIC) were performed (lower panels). The amount of target cDNA was calculated from the $\mathrm{x}$-axis value at which log (signal cDNA / signal PCR MIMIC) becomes zero. 

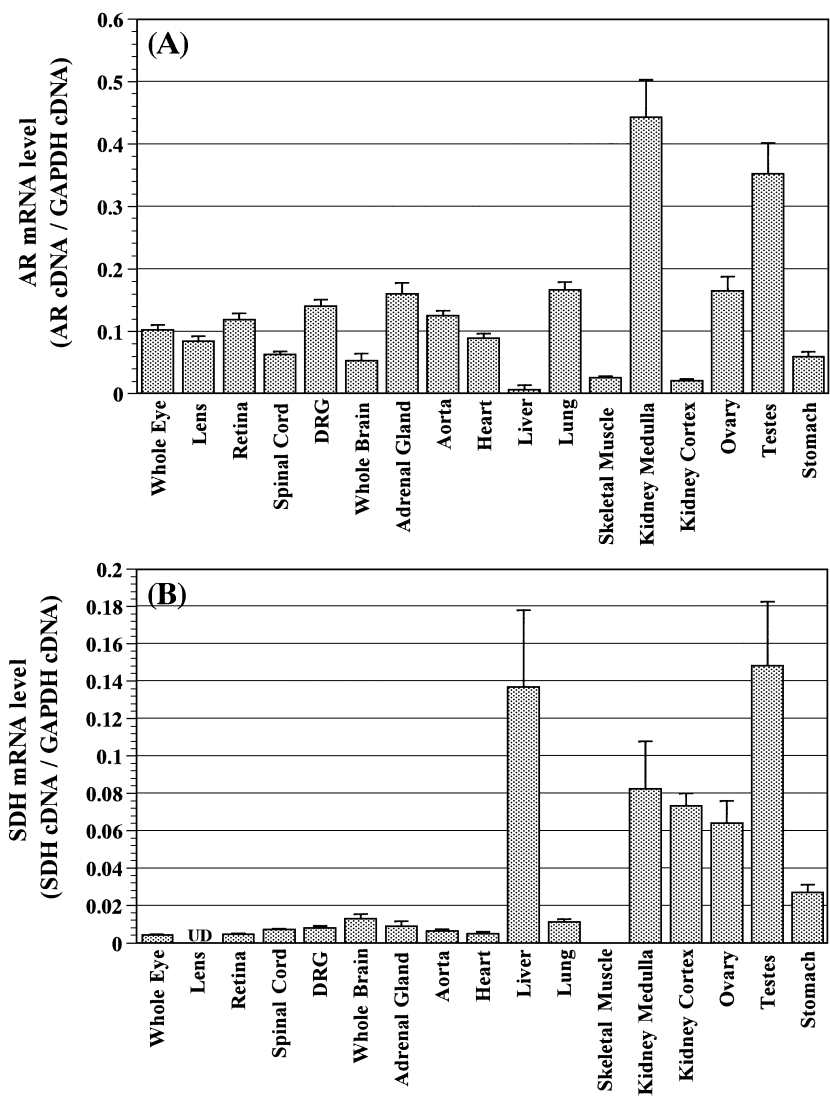

Fig. 2. AR (aldose reductase, A) mRNA and SDH (sorbitol dehydrogenase, B) mRNA expression in various rat tissues. The levels of mRNA are represented relative to GAPDH (glyceraldehyde3 -phosphate dehydrogenase) and are shown as the mean \pm S.E.M. of four separate experiments, each with the RNA pool taken from three rats. DRG: dorsal root ganglia, UD: under detection limit.

in semen (6), respectively. It is noteworthy that the target tissues for diabetic complication such as retina, lens, whole eye, aorta, heart and Schwann cells showed the same moderate expression of AR mRNA. Although the regulation of AR expression in these tissues under diabetic conditions has not been clearly elucidated, it may be involved in the pathogenesis of diabetic complications such as retinopathy, cataract, macroangiopathy, cardiomyopathy and neuropathy. In other tissues, where the relationship of AR to diabetic lesions is unclear, the expression levels of AR may be related to a physiological role of this enzyme such as detoxification of endogenous toxic aldehydes (7).

SDH mRNA was detected in all tissues investigated except lens (Fig. 2B). SDH mRNA was most abundant in testes and liver, while large amounts were also present in kidney medulla, kidney cortex and ovary. Low levels of expression were observed in stomach, whole brain and lung. Much lower levels were observed in adrenal gland, dorsal root ganglia, spinal cord, aorta, heart, retina and whole eye. The skeletal muscle (gastrocnemius muscle)

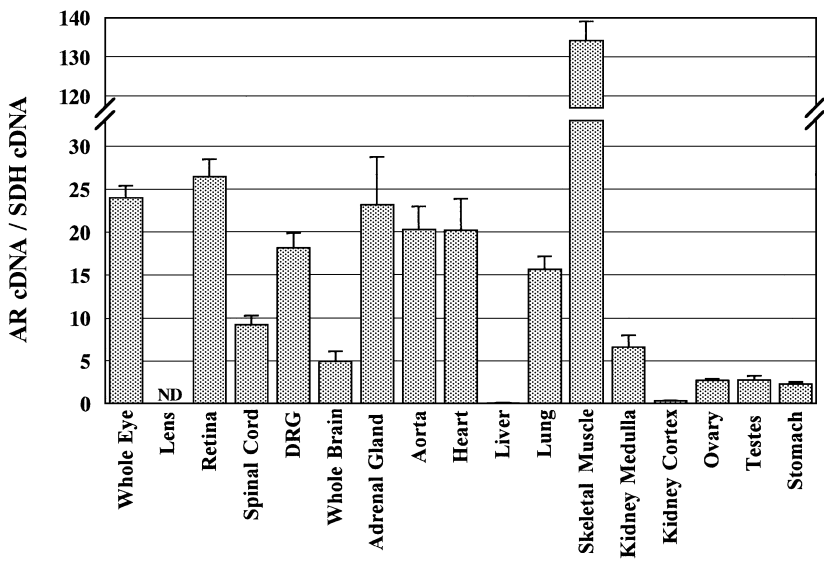

Fig. 3. Estimation of the ratio of AR (aldose reductase) cDNA to SDH (sorbitol dehydrogenase) cDNA in various rat tissues. Data are shown as the mean \pm S.E.M. of four separate experiments, each with the RNA pool taken from three rats. DRG: dorsal root ganglia, ND: not determined.

showed the lowest levels of mRNA, roughly 700-fold less than in testes. SDH mRNA was also expressed in the cultured rat Schwann cells, and its level was 0.01 as $\mathrm{SDH}$ cDNA / GAPDH cDNA ratio.

The high levels of SDH mRNA detected in testes, liver and kidney are in agreement with the previous reports on the expression profiles of rat (8) or mouse (9) SDH mRNA by Northern blot analysis. SDH, in conjunction with $\mathrm{AR}$, is thought to be involved in osmoregulation in kidney (5) and fructose synthesis in testis (6). In liver, it converts dietary sorbitol to fructose for further metabolism.

The level of SDH mRNA in rat lens was under the detection limit by our competitive RT-PCR assay. In human lens, SDH mRNA was expressed at the same level as in kidney (10), while in mouse lens, SDH mRNA expression was low by Northern blotting and not detectable by in situ hybridization (9). In addition, the enzyme activity of SDH in normal human lens has been observed to be higher than that in rat, rabbit and calf lens (11), further suggesting that the level of steady-state mRNA in rat lens is appreciably low compared to human lens. SDH mRNA expression in rat lens is minimal, and this may be the reason why opacification of lenses are more predominant in rats than in human under hyperglycemia.

We subsequently estimated AR cDNA/SDH cDNA ratios to compare the relative abundance of AR to SDH expression between various tissues of the rat (Fig. 3). The highest AR cDNA/SDH cDNA ratio was observed in skeletal muscle (gastrocnemius muscle), which is due to the lowest $\mathrm{SDH}$ expression in this tissue. This ratio in retina, whole eye, adrenal gland, aorta, heart, dorsal root ganglia and lung ranged from 10 to 30 , indicating that $\mathrm{AR}$ cDNA is 10 to 30 -fold higher than SDH cDNA. This ratio was 2 to 9 
in spinal cord, kidney medulla, whole brain, testes, ovary and stomach, while it was less than 1 in kidney cortex and liver, indicating that SDH cDNA is higher than AR cDNA. The ratio of AR cDNA to SDH cDNA in some tissues obtained by our competitive RT-PCR assay correlated well with the ratio of AR activity to SDH activity previously reported. For example, AR activity in rat liver was reported to be about one fiftieth of that of SDH (12), while the AR cDNA / SDH cDNA ratio was 0.04 by our competitive RT-PCR. In testes and whole brain, AR activity was reported to be about 6 to 7 times as much as that of SDH (13), while the AR cDNA/SDH cDNA ratio was 3 and 5, respectively. In rat Schwann cells, AR activity was about 4 to 5 times as much as that of SDH (T. Suzuki et al., unpublished observation), while the AR cDNA / SDH cDNA ratio in rat Schwann cells was 12 in our experiments. The AR cDNA / SDH cDNA ratios obtained by our competitive RT-PCR assay seem to reflect the relative abundance of AR to SDH expression, and they are useful to characterize the polyol pathway activity in various tissues.

In conclusion, the present study shows that different tissues contain varying amounts of AR mRNA and SDH mRNA; that is, each tissue has its own polyol pathway activity. It remains unknown whether the expression of these two enzymes and the relative abundance of SDH to $\mathrm{AR}$, which is shown to be peculiar to each tissue, fluctuate under diabetic conditions. These studies are helpful to ascertain the relative contributions of $\mathrm{AR}$ and $\mathrm{SDH}$ to the metabolic derangement resulting from the acceleration of polyol pathway activity in the target organs of diabetic complications.

\section{REFERENCES}

1 Gabbay KH: The sorbitol pathway and the complications of diabetes. N Engl J Med 288, 831 - 836 (1973)

2 Yabe-Nishimura C: Aldose reductase in glucose toxicity: a potential target for the prevention of diabetic complications. Pharmacol Rev 50, 21 - 33 (1998)

3 Maekawa K, Tanimoto T, Okada S, Suzuki T, Suzuki T and Yabe-Nishimura C: Expression of aldose reductase and sorbito dehydrogenase genes in Schwann cells isolated from rat: effects of high glucose and osmotic stress. Mol Brain Res 87, 251 - 256 (2001)

4 Suzuki T, Mizuno K, Yashima S, Watanabe K, Taniko K, Suzuki $\mathrm{T}$ and Yabe-Nishimura $\mathrm{C}$ : Characterization of polyol pathway in Schwann cells isolated from adult rat sciatic nerves. J Neurosci Res 57, 495 - 503 (1999)

5 Burg MB: Role of aldose reductase and sorbitol in maintaining the medullary intracellular milieu. Kidney Int 33, 635-641 (1988)

6 King TF and Mann T: Sorbitol metabolism in spermatozoa. Proc R Soc Lond Biol Sci 151, 226 - 243 (1959)

7 Srivastava SK, Chandra A, Srivastava S, Petrash JM and Bhatnagar A: Regulation of aldose reductase by aldehydes and nitric oxide. Adv Exp Med Biol 463, 501 - 507 (1999)

8 Estonius M, Danielsson O, Karlsson C, Persson H, Jornvall H and Hoog JO: Distribution of alcohol and sorbitol dehydrogenases. Assessment of mRNA species in mammalian tissues. Eur J Biochem 215, 497 - 503 (1993)

9 Lee FK, Lee AYW, Lin CXF, Chung SSM and Chung SK: Cloning, sequencing, and determination of the sites of expression of mouse sorbitol dehydrogenase cDNA. Eur J Biochem 230, 1059 - 1065 (1995)

10 Iwata T, Popescu NC, Zimonjic DB, Karlsson C, Hoog JO, Vaca G, Rodriguez IR and Carper D: Structural organization of the human sorbitol dehydrogenase gene (SORD). Genomics 26, $55-62$ (1995)

11 Jedziniak JA, Chylack LT, Cheng HM, Gillis MK, Kalustian AA and Tung WH: The sorbitol pathway in the human lens: aldose reductase and polyol dehydrogenase. Invest Ophthalmol Vis Sci 20, 314 - 326 (1981)

12 Gaynes BI and Watkins JB: Comparison of glucose, sorbitol and fructose accumulation in lens and liver of diabetic and insulintreated rats and mice. Comp Biochem Physiol [B] 92, 685 - 690 (1989)

13 Kicic E and Palmer TN: Is sorbitol dehydrogenase gene expression affected by streptozotocin-diabetes in the rat? Biochim Biophys Acta 1226, 213 - 218 (1994) 